\title{
Fire Image Set for Evoking Panic
}

\section{Iqbal Sabilirrasyad, Achmad Basuki, Tri Harsono}

\author{
Department of Information and Computer Engineering \\ Graduate Program of Engineering Technology \\ Politeknik Elektronika Negeri Surabaya \\ Jl. Raya ITS, Sukolilo, Surabaya, 60111, Indonesia \\ Telp: 62315947280 Fax: 62315946114 \\ E-Mail : iqbalnorth@gmail.com, basuki@pens.ac.id, \\ trison@pens.ac.id
}

Received May 15, 2020; Revised July 22, 2020; Accepted October 11, 2020

\begin{abstract}
Fire is the closest disaster to us, a person who put cigarettes around flammable objects could burn one to dozens of houses. The last thing that happens was a mass panic. In this kind of situation, panic is one of the keys to determine how much probability someone will survive. However, detecting someone's panic during a fire is impossible. This leads some scientists to assume that mass panic was never happening and some studies use simple functions to determine someone when panic. Currently, thanks to technological advances we can easily build virtual worlds that resemble real events. To build a virtual world that could evoke panic we still need the right stimulus. In this paper, we will discuss with terms of fire disaster stimulus that possible to impel someone to feel panic. While some stimulus datasets that already exist have more broad categories, we wanted to focus on a specific problem. The determined parameters are considered through several elements that could cause a person to panic, either before or during a fire. By using the Self-Assessment Manikin system to obtain valance and arousal matrix, we conduct a test to see how much influence the fire categories stimulus provided.
\end{abstract}

Keywords: Self - Assessment Manikin, Panic, Fire.

\section{INTRODUCTION}

Panic is a natural response possessed by living beings when they see, hear or feel what they fear. While we know not everything that is feared will make someone panic and not everyone has the same thing to scare about. Some people will afraid when they see snakes, but some will not, or even sometimes some people will panic over trivial things. We can find out someone is having a panic or not through some symptoms such as an increased heart rate, sweating, or feeling nausea [1]. However, in reality, it is impossible to check or detect symptoms experienced when a disaster occurs. Sometimes it's just a second perception from someone else saw us looks like 
having panic, or even the result of the media's exaggerated. In some cases when a fire broke out, instead of flight people more tend to have affiliation and trying to warn others [2]. But on the other hand, we know we can feel panic whenever and wherever even while in danger.

The danger of panic is explained by Quaranteli [3] is panic while in flight conditions. in this condition usually people whom panic will run blindly, which could lead the person to run toward the source of danger or a dead end instead. For example in a house fire when someone dealing with thick smoke and the person is in a state of panic. he could rush toward the smoke and end up into a dead-end or suffocate from lack of oxygen. Of course, not everyone will panic when they see thick smoke or blazing fire in front of them.

In the US In 2018 it was recorded that 2,720 people died and 11,200 people were injured in fires. And in the last five years, there have been 354,400 cases of house fires. The sources of the fire come from stove, heater, electricity, and cigarettes. A fire disaster is the closest disaster to the community, this is due to the ease with which a house fire occurs. Fire is used as a case in this research because it has the highest probability of death caused by carelessness when in panic. Fire also has the same conditions as described by Quaranteli needed for panic to occur [2]. If we use the conditions given by Quranteli to determine if a person is panicking in a fire, it is impossible that we receive what we wanted. It is clear in this state panic is still a black box.

In 1965 a simulation models are built to evoke panic when in evacuation conditions. Harold, et al [4] build a room to perform a test on the subject to escape from the given room. Some will be given a penalty when they fails. This punishment is believed to provide a stimulus to arouse panic when trying to escape. As the penalty for failing to escape from the room was increased, the success rate was found to be decreased. Because of this punishment, people will feel more fear and panic toward the punishment not because of what is happening around them. But this does not rule out the possibility of panic in a state of life risk. Unfortunately, we cannot use the same method while disaster occur. Some more specific parameter is needed to able describe the elements that can make someone panic.

If we backtrack to the fundamental definition of panic. Panic will occur caused by excessive fear. By intensifying fear we hoped that it could increase the possibility that someone will panic [1]. One way to arouse fear is through stimulus. This stimulus can originate from interaction, visual, and sound. Visual stimulus is the most difficult stimulus to build compared to other stimuli. Nowadays the type of stimulus for visual has been expanded, where one of them is the international affect picture system (IAPS) [5]. IAPS is a database that provides a set of images with emotional stimuli. As for similar databases such as the Geneva affective picture database (GAPED) [6], and the Open Affective Standardized Image Set (OASIS) [7]. Although there are many different kinds of databases, the categories given are still broad in 
characteristics. Specific categories such as fire or natural disasters is not significant. So it is difficult to define what kind of fire or how thick the smoke that can cause someone to panic.

The similarity of the three databases mentioned from IAPS, GAPED, and OASIS is using the same matrix, which is valance and arousal to determine the value of a stimulu. Self-assessment manikin (SAM) system [8] was used to attain valance and arousal matrix. SAM is a non-verbal pictorial assessment technology that directly measures the valence, arousal, origin, dominance, source, and significant associated with a person's affective reaction to a wide variety of stimuli. SAM has a scale of 1-9 with Valance is someone's impression of the object he sees. Arousal lowest scale for something calming and the highest scale more like bursting arousal. As for some other parameters such as origin, dominance, source, and significant are not used since the valance and arousal matrix is sufficient to determine a picture stimuli.

In this paper we proposed a set of visual stimulus that is specific for fire hazards. Provided images will be divided into several categories and several sub-categories related to the fire event. By using self-assessment manikin system to obtain valance and arousal data, we conduct a test to see how much influence parameter provides stimulus.

\section{RELATED WORKS}

The importance of using parameters as foundation used so the simulation is not deviated from the research subject. Not to mention that to build a system that is very detailed and able to provide stimuli, sufficient parameters is needed. In research conducted by Dan Liao, et al [9] building a virtual reality to arouse fear. The parameters used are visual stimulus database, sound, and video. By taking the arousal matrix value $>4$ and use it as a parameter to build virtual reality. From the results of grouping parameters, there are three scenes formed. These scenes are built from the parameters of location, elements, color, lighting, dynamic effects, plot, rhythm, sound, sound effect, and perspective camera. Two of the three scenes provided uses zombie as the main element in the scene, while the other scenes use insects. In this case, the use of broad categories from database can still be allocated but when building specific systems it requires specific parameters as well. The lack of detail about the categories needed to build a simulation is able to produce different interpretations of the simulation results.

\section{ORIGINALITY}

This study provides a set of images to narrow the existing database. Where in previous studies using broader categories such as Animal, Object, Person, Scenery. In this study, the image set was narrowed into some categories which include the fire hazard parameter. These categories are fire behaviours, fire sizes, smoke thickness, sources of fire, causes of death. Each 
category later divided by a sub-categories. By using the same method of selfassessment manikin as other databases, the image set provided is expected to be a specific stimulus in the case of fire.

\section{SYSTEM DESIGN}

In this section, the system design will be explained up to the process of analyzing the results in the proposed research.

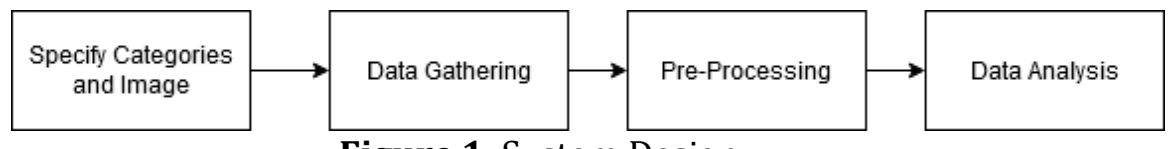

Figure 1. System Design

\section{A. Specify Categories and Images}

The categories selected based through several parameters that are able to change the conditions of events that occur up to a parameter considered to be able to change person responses when they see it directly. The first category is a behavior of fire, which consist of controlled fire and uncontrolled fire. Controlled fire is interpreted as fires that are easily regulated by the level of fire or easy to put out by the average people. Whereas uncontrolled fire interpreted as fires are difficult to extinguish unless an experienced person handles it.

The second category is the size of the fire. this parameter will be used to deduct the result from the previous category. Mainly this category focuses on uncontrolled fire and divided into two sub-categories large (at least the size of the house), and small (as high as an adult).

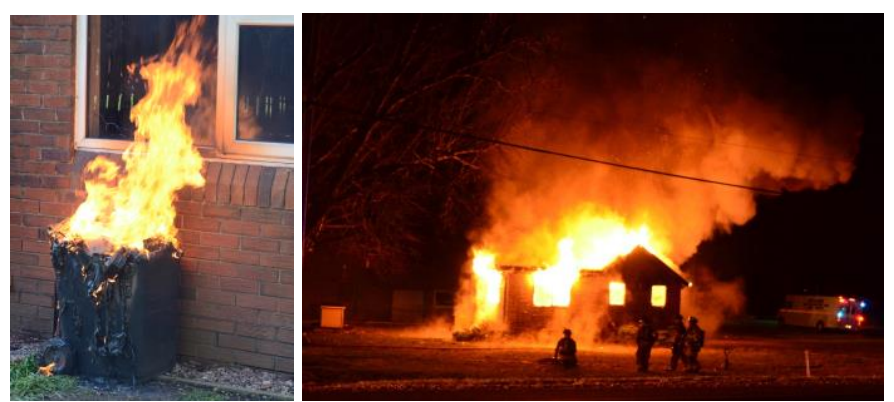

Figure 2. Small size fire (left) and big size fire (right).

Apart from the fire in general, there are several elements to consider such as smoke. In the book of Fire Toxicity [9], it is explained that the danger of fire smoke takes more lives than the fire itself. In addition, smoke is the first one to identify when there is a real fire occurs. Under conditions like watching fire hazards through different buildings or a safe distance. it's very rare to see people who panic because they still have a lot of time to prepare themselves. While in a different situation, like when we are in the same building as the fire occurs, the thickness of the smoke is able to give the perception of how big the fire has devoured the building. There is a $30 \%$ 
probability chance someone turn back the thickness of the smoke is $>0.3$ $\mathrm{OD} / \mathrm{m}$ [9]. But even so some people can tell how dangerous the condition and some people can't. For some people who can't tell the difference, this could lead to a deceiving perception and could lead someone into a panic state. We dicided to include smoke parameter into already existed parameters. In the third category, smoke will be divided into two, thin and thick. Where thin smoke still can give vision, and thick smoke can barely give any vision.

The fourth category is the source of the fire. Earlier we talk the most common and nearest community disaster was a house fire. This category will mainly be focused on how people will react to a fire starter. According to the firefighter website, there are four most common types of fires. These four types will be used as a sub-categories, among others are kitchen fires, electrical, heater, smoking-related fire.

The fifth category is the cause of death, which is by smoke and fire. These categories were addressed to know how people will react to related or someone else that was found dead. From five categories mentioned before we have a total of 12 sub-categories. Each sub-category will have 3 images to represent each sub-category. With a total overall picture is 36.

\section{B. Data Gathering}

Data gathering was collected by using the SAM system using a webbased questionnaire(Figure 3). Where participants will be asked to fill the point that will represent arousal and valance from the presented picture. The pictures provided are not shuffled but in through each sub-category 3 times (number of images in each sub-category). Before starting to fill in the questionnaire, the subject was described what valance and arousal matrix was, also what feeling they represent. The subject of the questionnaire ranges from 17-40 years old.

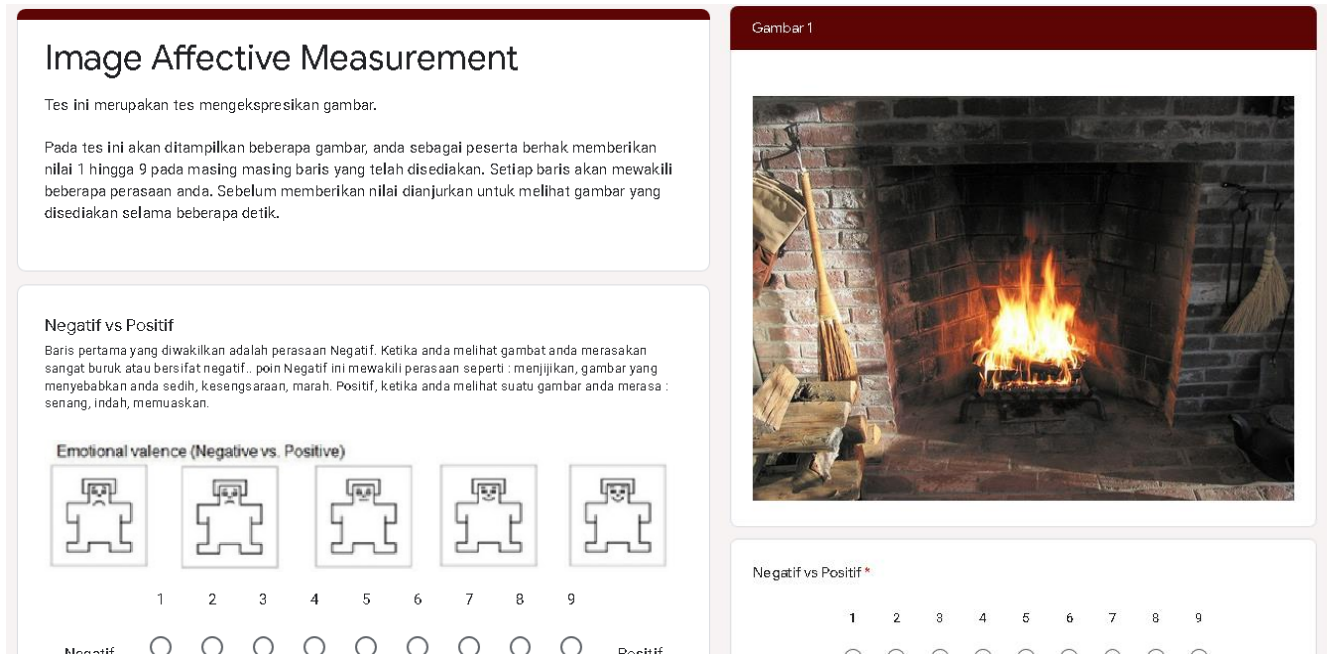

Figure 3. Picture of questionnaire website 


\section{Pre-processing}

In this process, the data that been obtained will be pre-processed by obtaining the average of each category and sub-categories. Also, the data will be separated based on the category of gender.

\section{Data Analysis}

In this process, the result will be analyzed in each subcategory using ANOVA and correlation between subcategories.

\section{EXPERIMENT AND ANALYSIS}

Data obtained with a total of 67 respondents ( 16 women and 49 men). the test is assessed on a scale of 1-9 for valence and arousal on all subcategories. As the result shown in table 1 the average valence of the whole dataset is 3.47 with $\mathrm{SD}=2.34$ and the mean arousal is 3.4 with $\mathrm{SD}=$ 2.514966. The standard deviation achieved is higher than expected, not to mention the number of dataset images provided is not too much (a total of 36). Although the average valence and arousal results obtained are close to the available image database values in the fire category.

Table 1. Dataset mean, standar deviation, emdian summary

\begin{tabular}{|c|c|c|c|c|c|c|c|}
\hline \multirow{2}{*}{ subcategories } & \multicolumn{3}{|c|}{ Valence } & \multicolumn{3}{|c|}{ Arousal } & \multirow{2}{*}{ categories } \\
\hline & mean & sd & median & mean & sd & median & \\
\hline \multirow{3}{*}{$\begin{array}{l}\text { controllable } \\
\text { uncontrollable } \\
\text { big }\end{array}$} & 6,79 & 1,80 & 7 & 6,02 & 2,30 & 6,50 & \multirow{12}{*}{$\begin{array}{l}\text { behaviour } \\
\text { behaviour } \\
\text { size } \\
\text { size } \\
\text { smoke } \\
\text { smoke } \\
\text { source } \\
\text { source } \\
\text { source } \\
\text { source } \\
\text { death } \\
\text { death }\end{array}$} \\
\hline & 2,78 & 2,00 & 2 & 5,01 & 2,66 & 5,00 & \\
\hline & 3,02 & 2,01 & 3 & 4,82 & 2,32 & 5,00 & \\
\hline \multirow{9}{*}{$\begin{array}{l}\text { small } \\
\text { thick } \\
\text { thin } \\
\text { cigarette } \\
\text { electricity } \\
\text { heater } \\
\text { stove } \\
\text { fire } \\
\text { smoke }\end{array}$} & 3,14 & 1,77 & 3 & 4,52 & 2,29 & 5,00 & \\
\hline & 4,07 & 2,48 & 4 & 4,79 & 2,35 & 5,00 & \\
\hline & 4,69 & 2,17 & 5 & 4,74 & 2,06 & 5,00 & \\
\hline & 3,27 & 2,16 & 3 & 3,99 & 2,42 & 4,00 & \\
\hline & 2,49 & 1,57 & 2 & 4,56 & 2,64 & 4,00 & \\
\hline & 3,94 & 2,11 & 4 & 4,71 & 2,13 & 5,00 & \\
\hline & 2,76 & 2,02 & 2 & 4,78 & 2,64 & 5,00 & \\
\hline & 1,97 & 1,73 & 1 & 4,22 & 3,04 & 4,00 & \\
\hline & 2,74 & 1,91 & 2 & 3,83 & 2,35 & 4,00 & \\
\hline total image & 3,47 & 2,34 & & 4,67 & 2,51 & & \\
\hline
\end{tabular}

From Table 1. The highest valence value from subcategories is the first subcategory (controllable fire) with a mean value of 6.79. which is no surprise. Since theoretically, the image provided should enter the positive region. For the second and third highest mean, happen in the smoke category. With sub-category thin smoke with a mean value of 4.69 and thick smoke mean value of 4.07. What's interesting controlled fires mostly considered positive or some people more likely tell its positive stimuli. If we look back to the intentional purpose of using the sub-category of controlled fire and uncontrolled fire. We can conclude that generally, people are not afraid of fire. But the feeling of cannot control the fire and the fact fire also able to harm us change fire into a negative stimulus. From the results, the smallest 
valence obtained with a mean value of 1.97 in the sub-category of death due to fire, which theoretically should be in the negative section. Whereas the highest value for arousal with a mean value of 6.02 is controlled fire subcategory. While for the lowest with a value of 3.83 in the category of death due to smoke. In theory, the largest arousal value obtained is not expected to be in the category of controlled fire. Where the positive value on the valence obtained.

We also conducted a one-way ANOVA analysis to see if there any significant differences. ANOVA is performed in each mean on the subcategories. With the null hypothesis, the mean difference between subcategories is significant for both valence and arousal data.

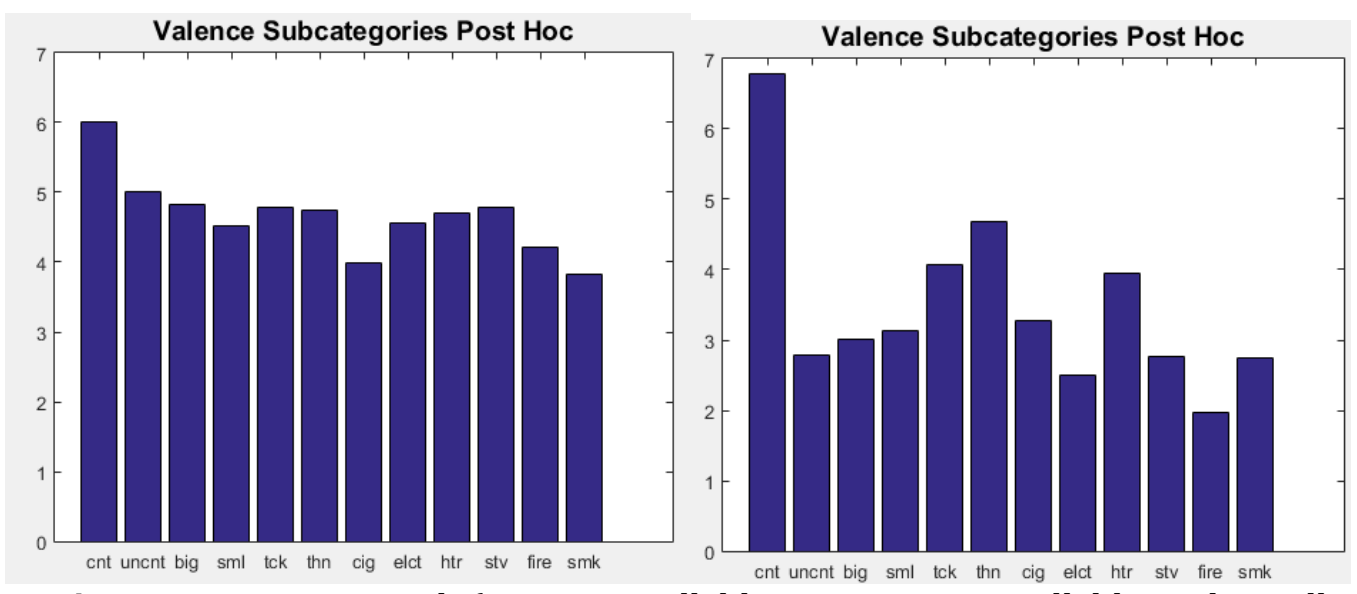

Figure 4. Post Hoc result (cnt = controllable; uncnt=uncontrollable; sml=small; tck=thick; thn=thin; cig=cigarette; elct=electricity; htr=heater; stv=stove; smk=smoke; $\alpha=0.05$ )

Anova's results for Valence and arousal invalidate the initial hypothesis. The value obtained is mean valence $F(11.2364)=81.73, p<0.01$. and for arousal where $F(11.2364)=10.03, p<0.01$. We can conclude from the ANOVA results for arousal and valence, that there are significant differences between subcategories. From the ANOVA test, we have found that there are differences from the average between groups. Then we perform post hoc using Tukey Kramer HSD from subcategories.

As the result of post hoc (figure 4), some categories diverge significantly and there are several adjacent categories. What's interesting is that several subcategories have very significant differences in their category. As in the category of fire behavior which consists of controlled fire and uncontrollable fire. The two subcategories have distinct differences (controllable $=6.7929$, uncontrollable $=2.7778$ with $\mathrm{SD}=0.1421$ ). For subcategories of large fires with a value of 3.0202 and small fires with a value of 3.1414 have the same level of similarity in one category. As for arousal post hoc results of arousal, almost all of the existing subcategories have a high degree of similarity, except for arousal in the fire subcategory of controlled fire with a value of $=6.0202$. 


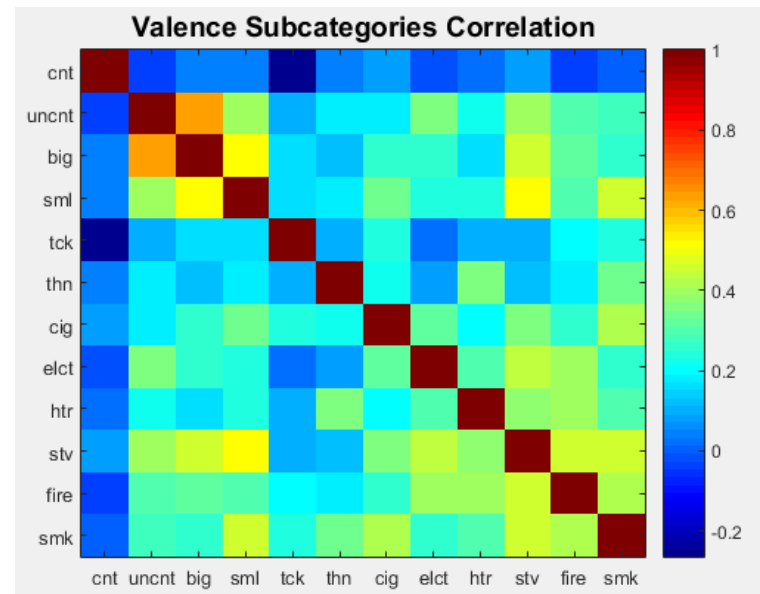

Figure 5. Correleation subcategories heat map ( $\mathrm{cnt}=$ controllable; uncnt=uncontrollable; sml=small; tck=thick; thn=thin; cig=cigarette; elct=electricity; htr=heater; stv=stove; smk=smoke; $\alpha=0.05$ )

We also examine correlations (figure 5) from each of the existing subcategories. From several subcategories, there can be developed a scenario that theoretically can enhance the level of stimulus. Correlations are sought based on the average valence of each subcategory. The results of the correlation between the subcategory obtained are not that high. The results from Figure 4 where the highest correlation value is the correlation between the uncontrolled fire sub-category with a large fire $(p=0.6361)$. Where the relationship between the two subcategories is quite interesting. When we talk about aspects in the fire we do not know which elements are able to provide good stimuli. Clearly, people will afraid when dealing directly with a big uncontrollable fire, but we do not know whether all people who deal with similar things will feel fear. But in the statistical results of this test, we manage to conclude that most people are afraid of it. The second-largest correlation value is $p=0.5131$, where a correlation between the source fire originating from the stove and medium-sized fire with a value. The relationship between subcategories with medium fire with high fire received the third-highest value with $\mathrm{p}=0.5103$.

\section{CONCLUSION}

In the statistical results, if seen through the entire dataset we can be assumed that in general fire has a negative stimulus. But in the post hoc result, it shows that the subcategory for controlled fire with the highest average value gives a different value than the other subcategories. This category is taken with the assumption to find out whether human beings are generally afraid or have negative value to fire or not. Finally, the results of the existing statistical data confirm in the area of research for sub-categories, most people who are not generally afraid of fire.

From the observations of the correlation made the results obtained on average are not too high. But the results of the analysis of the correlations 
yielded some interesting matches for the existing subcategories. The relationship between these subcategories is theoretically able to provide more stimulus compared to the attributes that stand alone.

From the fire image dataset provided gives a fairly low valence with value of 3,47 and rousal with value of 4,67 . Which can be interpreted as considerably scary. From this research we know fire has a potential to be a panic stimulus. But to be able to make someone panic still need a consistent fear stimulus. The use of existing datasets can be considered by providing some other media such as audio or interaction to increase the existing stimulus.

\section{REFERENCES}

[1] L. Marsh, Anxiety and panic attacks, Mind, pp. 1-26, 2015, doi: 10.1007/s00259-002-0914-2.

[2] E. A. Der Heide, Common Misconceptions about disasters: Panic, the "Disaster Syndrome," and Looting. 2004.

[3] E. L. Quarantelli, Panic Behaviour: Some Empirical Observations, Hum. Response to Tall Build., pp. 336-350, 1975.

[4] H. H. Kelley, J. C. Condry, A. E. Dahlke, and A. H. Hill, Collective behavior in a simulated panic situation, J. Exp. Soc. Psychol., vol. 1, no. 1, pp. 20-54, 1965, doi: 10.1016/0022-1031(65)90035-1.

[5] E. K. B. Verschuere, G. Crombez, The international affective picture syst Flemish.pdf. pp. 205-217, 2001.

[6] E. S. Dan-Glauser and K. R. Scherer, The Geneva affective picture database (GAPED): A new 730-picture database focusing on valence and normative significance, Behav. Res. Methods, vol. 43, no. 2, pp. 468-477, 2011, doi: 10.3758/s13428-011-0064-1.

[7] B. Kurdi, S. Lozano, and M. R. Banaji, Introducing the Open Affective Standardized Image Set (OASIS), Behav. Res. Methods, vol. 49, no. 2, pp. 457-470, 2017, doi: 10.3758/s13428-016-0715-3.

[8] M. M. Bradley and P. J. Lang, Measuring emotion: The selfassessment manikin and the semantic differential, J. Behav. Ther. Exp. Psychiatry, vol. 25, no. 1, pp. 49-59, 1994, doi: 10.1016/00057916(94)90063-9.

[9] M. Wallmyr, D. Kade, and T. Holstein, Virtual, Augmented and Mixed Reality: Applications in Health, Cultural Heritage, and Industry, vol. 10910. Springer International Publishing, 2018.

[10] M. Ziky, Review of $A^{*}$ (A Star) Navigation Mesh Pathfinding as the Alternative of Artificial Intelligent for Ghosts Agent on the Pacman Game, doi: 10.24003/emitter.v4i1.117 